\title{
Passive bilateral leg cycling with concomitant regional circulatory occlusion for testing mechanoreflex-metaboreflex interactions in humans
}

\author{
Adrian Lis $^{1}$ (D) $\cdot$ Wojciech Łopusiewicz ${ }^{1}$ (D) Massimo F. Piepoli ${ }^{2,3}$ (D) $\cdot$ Beata Ponikowska $^{1}$ (D) Bartłomiej Paleczny ${ }^{1}$ (D)
}

Received: 18 January 2020 / Accepted: 30 July 2020 / Published online: 8 August 2020

(c) The Author(s) 2020

\begin{abstract}
Purpose The exercise pressor reflex (EPR) plays a fundamental role in physiological reactions to exercise in humans and in the pathophysiology of cardiovascular disorders. There is no "gold standard" method for EPR assessment; therefore, we propose a new protocol for testing interactions between the muscle mechanoreflex and metaboreflex (major components of EPR). Methods Thirty-four healthy subjects (mean age [ \pm standard deviation] $24 \pm 4$ years, 22 men) were enrolled in the study. During the study, the hemodynamic and ventilatory parameters of these subjects were continuously monitored using our proposed assessment method. This assessment method consists of an initial 5-min rest period (baseline) followed by 5 min of passive cycling (PC) on an automated cycle ergometer (mechanoreceptor stimulation), after which tourniquet cuffs located bilaterally on the upper thighs are inflated for $3 \mathrm{~min}$ to evoke venous and arterial regional circulatory occlusion (CO) during PC (metaboreceptor stimulation). Deflation of the tourniquet cuffs is followed by a second $5 \mathrm{~min}$ of PC and finally by a 5-min recovery time. The control test comprises a 5 -min rest period, followed by $3 \mathrm{~min}$ of $\mathrm{CO}$ only and a final 5-min recovery.

Results Mean arterial pressure (MAP) and minute ventilation (MV) increased significantly during PC (MAP: from $90 \pm 9.3$ to $95 \pm 9.7 \mathrm{mmHg}$; $\mathrm{MV}$ : from $11.5 \pm 2.5$ to $13.5 \pm 2.9 \mathrm{~L} / \mathrm{min}$; both $p<0.05$ ) and again when CO was applied (MAP: from $95 \pm 9.7$ to $101 \pm 11.0 \mathrm{mmHg}$; $\mathrm{MV}$ : from $13.5 \pm 2.9$ to $14.8 \pm 3.8 \mathrm{~L} / \mathrm{min}$; both $p<0.05)$. In the control test there was a slight increase in MAP during CO (from $92 \pm 10.5$ to $94 \pm 10.0 \mathrm{mmHg} ; p<0.05$ ) and no changes in the ventilatory parameters. Conclusion Bilateral leg passive cycling with concomitant circulatory occlusion is a new, simple and effective method for testing interactions between the mechanoreflex and metaboreflex in humans.
\end{abstract}

Keywords Exercise pressor reflex $\cdot$ Mechanoreceptors $\cdot$ Metaboreceptors $\cdot$ Passive cycling $\cdot$ Circulatory occlusion

Electronic supplementary material The online version of this article (https://doi.org/10.1007/s10286-020-00717-x) contains supplementary material, which is available to authorized users.

Adrian Lis

adrian.lis@student.umed.wroc.pl

1 Department of Physiology, Wroclaw Medical University, ul. Chałubińskiego 10, 50-368 Wroclaw, Poland

2 Department of Cardiology, Polichirurgico Hospital G Da Saliceto, Saliceto, Italy

3 Institute of Life Sciences, Sant'Anna School of Advanced Studies, Pisa, Italy

\section{Introduction}

The exercise pressor reflex plays a fundamental role in physiological responses to exercise in humans [1-4]. It is triggered by the stimulation of muscle metaboreceptors, specifically by the metabolites produced when muscles contract, and by muscle mechanoreceptors, through the mechanical distortion of contracting muscles [1-3], and leads to sympathetic activation and vagal withdrawal that typically manifests as increases in cardiac output and blood pressure $[1-3,5]$. These changes enable the human body to maintain perfusion pressure, thereby improving the blood supply to working muscles during exercise [6]. These effects, observed even in passive exercise, are reinforced during voluntary movements by the central mechanism, commonly known as "central command," which is a complex feedforward mechanism originating in the brain [7]. The contribution of central 
command is a confounding factor and therefore should be excluded in experiments investigating the isolated role of muscle mechano- and metaboreflexes. Overactivity of the exercise pressor reflex has been associated with certain cardiovascular disorders, such as arterial hypertension and heart failure, and linked to exaggerated sympathetic outflow and hyperventilation, which are characteristics of these diseases $[1,2,8-11]$.

Isolated responses from metaboreceptors can be measured with static exercise testing (e.g. handgrip test) and postexercise regional circulatory occlusion $[8,9]$, whereas the evaluation of muscle mechanoreflex sensitivity in humans remains a challenge. Several methods have been proposed, such as measuring passive movements [8, 14, 15], electrical stimulation $[9,16]$ and muscle stretching [17-20], but none of these are considered to be the "gold standard" due to inherent limitations. For example, the reproducibility of measurements of passive movements is a concern due to the measurements being performed by (different sets of) investigators or with custom-built equipment; electrostimulation evokes larger muscular metabolic perturbations than do voluntary movements [21-23]; and stretching muscles stimulate other types of mechanoreceptors than contracting muscles [1]. Even less is known about the interactions between the mechanoreflex and metaboreflex as very few studies have been performed [17-19, 24], all of which used the same muscle stretching protocol, and the results are highly discordant.

Here we propose a new method for testing the mechanoreflex-metaboreflex interactions in humans. The novelty of our method is that (1) it directly compares physiological responses to mechanoreceptor-only and mechanoreceptor-metaboreceptor activation; (2) it uses an exercise model resembling real-life activities (cycling); (3) it utilizes the "physiological" order of mechano- and metaboreflex stimulation (mechanoreceptors are stimulated first); and (4) it uses a commercially available, automated device for passive exercise (instead of custom-built devices or passive movements performed manually by a researcher). Although some of the aforementioned approaches considered separately are not novel (e.g. passive cycling [PC] has been used in an earlier study [14]), we are the first to address all of these factors simultaneously in a single experiment. The aim of this new strategy is to first induce isolated activation of the muscle mechanoreflex (using PC) and then to add metaboreflex stimulation (using circulatory occlusion [CO]) without stopping the PC so that both the mechano- and metaboreflexes are activated simultaneously. We hypothesized that PC alone would increase blood pressure and ventilation, that the subsequent addition of $\mathrm{CO}$ to $\mathrm{PC}$ would induce additional increases in these variables and that the values of these measures would return to the levels observed before PC and $\mathrm{CO}$ following cessation of these interventions.

\section{Methods}

\section{Subjects}

Thirty-four healthy volunteers participated in the study, of whom 22 were men. Mean age ( \pm standard deviation [SD]), height, weight and body mass index (BMI) of the subjects was $24 \pm 4$ years, $178 \pm 11 \mathrm{~cm}, 75 \pm 15 \mathrm{~kg}$ and $23.7 \pm 3.2 \mathrm{~kg} /$ $\mathrm{m}^{2}$, respectively. No previous histories of chronic disease were reported. The volunteers were asked to avoid intense exercise and drinking coffee for $24 \mathrm{~h}$ before the tests and eating food or smoking cigarettes for $2 \mathrm{~h}$ before the tests.

All subjects received detailed information about the study and gave written informed consent prior to participation. The protocol was approved by the local ethics committee. All procedures were performed according to the Declaration of Helsinki of 1964 and its later amendments.

\section{Experimental protocol and equipment}

The protocol consists of two tests: the main test and the control test (Fig. 1). During each test, hemodynamic and ventilatory parameters are continuously and noninvasively monitored and recorded. Heart rate (HR, in bpm) is calculated from lead II of the electrocardiogram (BioAmp; ADInstruments, Dunedin, New Zealand). Mean mean arterial pressure (MAP, $\mathrm{mmHg}$ ), systolic blood pressure (SBP, $\mathrm{mmHg}$ ) and diastolic blood pressure (DBP, $\mathrm{mmHg}$ ) are recorded at a sampling frequency of $250 \mathrm{~Hz}$ using the Nexfin device (BMEYE B.V., Amsterdam, The Netherlands) and

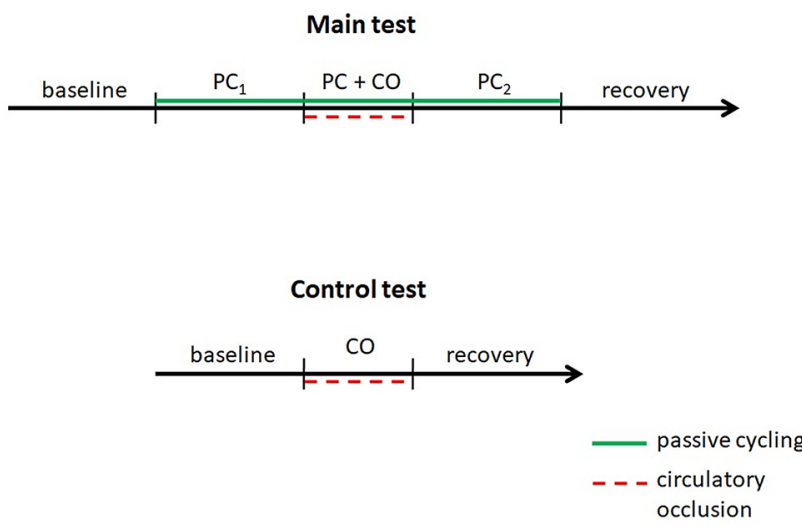

Fig. 1 The protocol consists of two tests (main test and control test) performed in a random order. The main test consists of five consecutive phases: a 5 -min resting period; $5 \mathrm{~min}$ of passive cycling $\left(P C_{I}\right)$; 3 min of circulatory occlusion during passive cycling $(P C+C O)$; a second 5 min of PC after cessation of $\mathrm{CO}\left(P_{2}\right)$; and a 5-min recovery period. The control test comprises three consecutive phases: a 5 -min resting period, followed by $3 \mathrm{~min}$ of $\mathrm{CO}$ and rounded off with a 5-min recovery period 
the volume-clamp method [25]. Stroke volume (SV, mL) and total peripheral resistance (TPR, dyn $\cdot \mathrm{s} \cdot \mathrm{cm}^{-5}$ ) are calculated from the recorded pressures using a pulse contour method; higher TPR units reflect stronger peripheral vasoconstriction. Minute ventilation ( $\mathrm{MV}, \mathrm{L} / \mathrm{min}$ ) is calculated from instantaneous values of the breathing rate (breaths/min) and tidal volume (L), measured using a differential pressure transducer (FE141 Spirometer; ADInstruments) and a breathing circuit consisting of an oronasal face mask (Hans Rudolph, Inc., Shawnee, KS, USA) and a two-way nonrebreathing T-shape valve (Hans Rudolph, Inc.) connected to a flowhead (MLT3000 L; ADInstruments) positioned on the expiratory arm of the breathing circuit. All data are recorded with an acquisition system (PowerLab; ADInstruments) at a sampling frequency of $1 \mathrm{kHz}$. To ensure that the central command was not engaged, we used a commercially available automated cycle ergometer (Medbike, BTL, UK) that is certified for use in rehabilitation of individuals with neural disorders.

The participants sit on the cycle ergometer with their knees flexed, their feet firmly attached to the pedals and their calves stabilized with rails connected to the pedals to prevent additional voluntary movements (Fig. 2). The distance between the seat and the ergometer is easily adjusted to ensure that there is a slight flexion in the knee when the foot is at its farthest point. The subjects are equipped with tourniquet cuffs attached bilaterally to their upper thighs during the entire protocol (Fig. 3). During the CO period of PC, the tourniquets are inflated $(200 \mathrm{mmHg})$ to trap metabolites in the lower limbs. The pressure level selected is based on similar previously reported experiments $[19,26]$, taking into account gravitational factors [27] and allowing for the occlusion of venous and arterial circulation without evoking pain.

The main test comprises five consecutive phases: (1) a 5-min resting period; (2) 5 min of PC on the ergometer, with

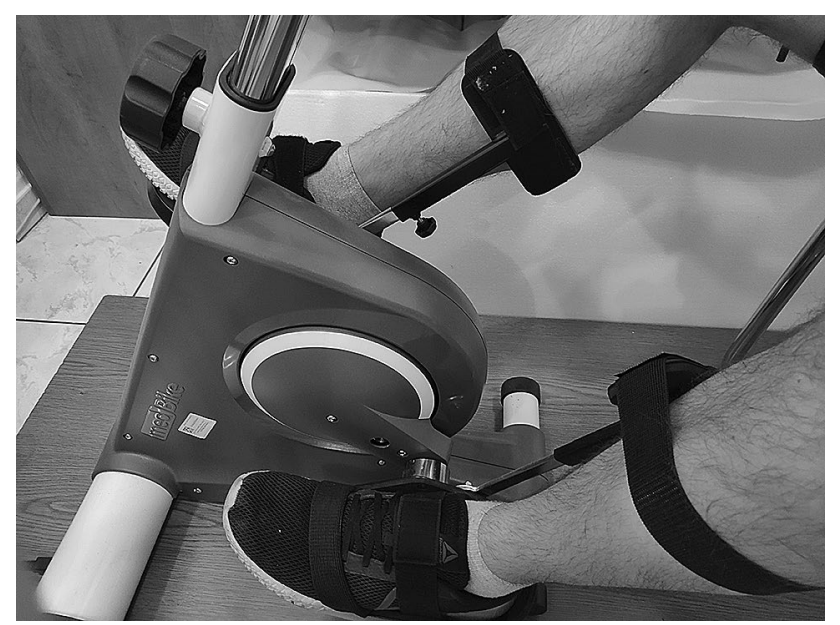

Fig. 2 Rails stabilizing a subject's feet

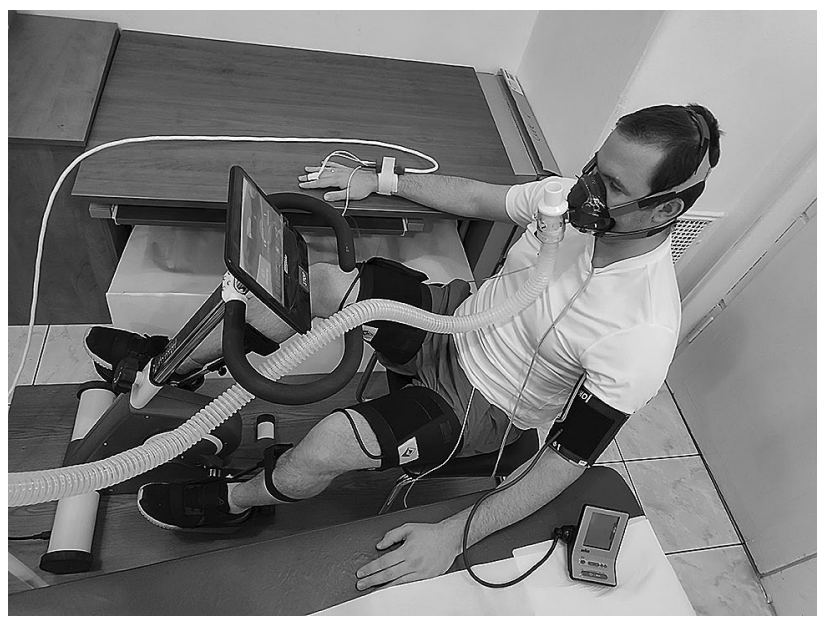

Fig. 3 Laboratory set-up

the pedaling rate set electronically at $60 \mathrm{rpm}$ (mechanoreflex activation); (3) 3 min of bilateral upper thigh tourniquet cuff inflation to evoke venous and arterial regional $\mathrm{CO}$ during PC (activation of mechanoreflex and metaboreflex); (4) deflation of tourniquets and an additional 5 min of PC (mechanoreflex activation); and finally (5) a 5-min recovery period after cessation of PC. Each subject is instructed to relax and not to use any muscles during the course of study in order to minimize central command contribution. The participants are observed throughout the protocol to ensure they do not contract their muscles voluntarily.

The control test is performed to ensure that $\mathrm{CO}$ does not evoke hemodynamic or respiratory changes due to, for example, psychological factors or compression-induced activation of the muscle mechanoreflex. It comprises three consecutive phases: (1) a 5-min resting period; (2) 3 min of bilateral upper thigh tourniquet cuff inflation; and (3) a 5-min recovery period.

The tests are performed in a random order during the same visit in our laboratory. All participants rest for $15 \mathrm{~min}$ before the procedures. After the experiment, the subjects are asked to assess the pain in their legs during cuff occlusion (0-10 scale) and perceived exertion during PC using a Borg scale (6-20 points) [28].

\section{Statistical analysis}

Data are presented as the mean \pm SD. All percentage changes were calculated as the difference between the values from the two periods divided by the value from the first period. Hemodynamic and ventilatory parameters from each part of the two tests were analyzed with repeated-measures analysis of variance (ANOVA) and Duncan's post hoc test. The two-way repeated-measures ANOVA was used to compare the differences between 
the main and control tests. $t$ tests were used for group comparisons. A $p$ value $<0.05$ was considered to be significant.

\section{Results}

All hemodynamic and ventilatory parameters collected during the main and control tests are summarized in Tables 1 and 2, respectively. Plots for the main test are

Table 1 Hemodynamic and ventilatory parameters recorded in the five consecutive phases of the main test

\begin{tabular}{|c|c|c|c|c|c|c|c|c|c|}
\hline \multirow{2}{*}{$\begin{array}{l}\text { Hemodynamic } \\
\text { and ventilatory } \\
\text { parameters }^{\mathrm{a}}\end{array}$} & \multicolumn{5}{|c|}{ Period of main test ${ }^{\mathrm{b}}$} & \multicolumn{4}{|l|}{$p$ value* } \\
\hline & $\begin{array}{l}\text { Baseline } \\
(5-\text { min rest- } \\
\text { ing period })\end{array}$ & $\mathrm{PC}_{1}$ & $\mathrm{PC}+\mathrm{CO}$ & $\mathrm{PC}_{2}$ & Recovery & $\begin{array}{l}\text { Baseline vs. } \\
\mathrm{PC}_{1}\end{array}$ & $\begin{array}{l}\mathrm{PC}_{1} \text { vs. } \\
\mathrm{PC}+\mathrm{CO}\end{array}$ & $\begin{array}{l}\mathrm{PC}+\mathrm{CO} \text { vs. } \\
\mathrm{PC}_{2}\end{array}$ & $\begin{array}{l}\mathrm{PC}_{2} \text { vs. } \\
\text { recovery }\end{array}$ \\
\hline MAP (mmHg) & $90 \pm 9.3$ & $95 \pm 9.7$ & $101 \pm 11.0$ & $96 \pm 9.8$ & $93 \pm 9.8$ & $<0.001$ & $<0.001$ & $<0.001$ & $<0.001$ \\
\hline $\mathrm{SBP}(\mathrm{mmHg})$ & $120 \pm 14.1$ & $130 \pm 14.4$ & $135 \pm 15.7$ & $131 \pm 14.6$ & $125 \pm 13.3$ & $<0.001$ & $<0.001$ & 0.002 & $<0.001$ \\
\hline DBP (mmHg) & $72 \pm 7.0$ & $75 \pm 7.4$ & $80 \pm 8.5$ & $75 \pm 7.4$ & $74 \pm 7.5$ & $<0.001$ & $<0.001$ & $<0.001$ & 0.02 \\
\hline $\begin{array}{l}\text { TPR } \\
\left(\text { dyn } \cdot \mathrm{s} \cdot \mathrm{cm}^{-5}\right)\end{array}$ & $1030 \pm 225$ & $952 \pm 194$ & $1003 \pm 219$ & $953 \pm 197$ & $1032 \pm 231$ & $<0.001$ & $<0.001$ & $<0.001$ & $<0.001$ \\
\hline $\mathrm{SV}(\mathrm{mL})$ & $96 \pm 16.1$ & $109 \pm 19.1$ & $104 \pm 19.7$ & $109 \pm 19.6$ & $100 \pm 15.6$ & $<0.001$ & $<0.001$ & $<0.001$ & $<0.001$ \\
\hline HR (bpm) & $76 \pm 11.2$ & $77 \pm 11.8$ & $81 \pm 13.6$ & $77 \pm 11.6$ & $75 \pm 10.8$ & 0.43 & $<0.001$ & $<0.001$ & $<0.001$ \\
\hline TV (L) & $0.80 \pm 0.3$ & $0.84 \pm 0.4$ & $0.93 \pm 0.5$ & $0.87 \pm 0.3$ & $0.80 \pm 0.3$ & 0.49 & 0.07 & 0.17 & 0.22 \\
\hline BR (bpm) & $15.3 \pm 3.6$ & $17.7 \pm 4.0$ & $17.3 \pm 3.8$ & $17.5 \pm 3.9$ & $15.4 \pm 3.5$ & $<0.001$ & 0.43 & 0.60 & $<0.001$ \\
\hline MV (L/min) & $11.5 \pm 2.5$ & $13.5 \pm 2.9$ & $14.8 \pm 3.8$ & $14.1 \pm 2.7$ & $11.4 \pm 2.6$ & 0.02 & $<0.001$ & 0.16 & $<0.001$ \\
\hline
\end{tabular}

Hemodynamic and ventilatory parameters in table are presented as the mean value \pm standard deviation (SD) for each consecutive phase of the mean test

*Changes in each parameter are statistically significant according to repeated measures analysis of variance (ANOVA), when all phases are considered together. Note: for TV only, baseline vs. PC $+\mathrm{CO}$ and PC $+\mathrm{CO}$ vs. recovery are statistically significant according to Duncan's post hoc test $(p=0.017$ and $p=0.014$, respectively)

${ }^{a} M A P$ Mean arterial pressure, $S B P$ systolic blood pressure, $D B P$ diastolic blood pressure, $T R P$ total peripheral resistance, $S V$ stroke volume, $H R$ heart rate, $T V$ tidal volume, $B R$ breathing rate, $M V$ minute ventilation

${ }^{b} \mathrm{PC}_{1}$ is the first passive cycling (PC) period; it follows baseline (the initial 5-min resting period) and is followed by passive cycling with circulatory occlusion $(\mathrm{PC}+\mathrm{CO})$. Once $\mathrm{CO}$ ceases, $\mathrm{PC}$ continues $\left(\mathrm{PC}_{2}\right)$. The last period is the recovery period. For full description, see section Experimental protocol and equipment

Table 2 Hemodynamic and ventilatory parameters recorded in the three consecutive phases of the control test

\begin{tabular}{|c|c|c|c|c|c|}
\hline \multirow{2}{*}{$\begin{array}{l}\text { Hemodynamic and } \\
\text { ventilatory parameters }\end{array}$} & \multicolumn{3}{|c|}{ Period of control test ${ }^{\mathrm{a}}$} & \multicolumn{2}{|l|}{$p$ value* } \\
\hline & Baseline & $\mathrm{CO}$ & Recovery & Baseline vs. CO & CO vs Recovery \\
\hline MAP, mmHg & $92 \pm 10.5$ & $94 \pm 10.0$ & $92 \pm 8.9$ & 0.001 & 0.001 \\
\hline SBP, mmHg & $123 \pm 14.7$ & $125 \pm 13.7$ & $123 \pm 11.8$ & 0.06 & 0.65 \\
\hline $\mathrm{DBP}, \mathrm{mmHg}$ & $73 \pm 8.1$ & $75 \pm 7.8$ & $73 \pm 6.9$ & $<0.001$ & 0.52 \\
\hline TPR, dyn $\cdot \mathrm{s} \cdot \mathrm{cm}^{-5}$ & $1024 \pm 216$ & $1061 \pm 218$ & $1035 \pm 220$ & $<0.001$ & 0.26 \\
\hline $\mathrm{SV}, \mathrm{mL}$ & $100 \pm 14.9$ & $99 \pm 15.1$ & $98 \pm 14.3$ & 0.45 & 0.70 \\
\hline HR, bpm & $76 \pm 10.1$ & $76 \pm 11.7$ & $75 \pm 11.2$ & 0.79 & 0.63 \\
\hline TV, L & $0.78 \pm 0.3$ & $0.86 \pm 0.4$ & $0.82 \pm 0.3$ & 0.10 & 0.46 \\
\hline $\mathrm{BR}, \mathrm{bpm}$ & $15.4 \pm 3.6$ & $15.6 \pm 3.5$ & $15.6 \pm 3.6$ & 0.67 & 0.71 \\
\hline $\mathrm{MV}, \mathrm{L} / \mathrm{min}$ & $11.2 \pm 2.3$ & $12.4 \pm 3.6$ & $11.9 \pm 2.8$ & 0.06 & 0.33 \\
\hline
\end{tabular}

Hemodynamic and ventilatory parameters in table are presented as the mean value \pm SD for each consecutive phase of the control test

*Changes in the SBP, SV, HR, TV, BR and MV are not significant statistically according to repeated measures ANOVA when all periods are considered together

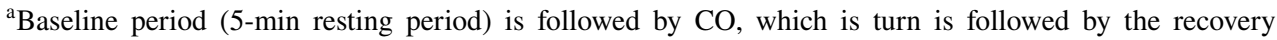
period. For full description, see section Experimental protocol and equipment 
depicted in Fig. 4. During the main test, all parameters exhibited significant changes from baseline when all periods were considered together (all ANOVA $p<0.001$ ). The changes in blood pressure and ventilation presented consistent patterns, including an initial increase in response to PC that increased further with $\mathrm{CO}$. A different response was observed in SV, which increased during PC and then decreased slightly with CO. TPR followed the opposite pattern, decreasing with $\mathrm{PC}$ and slightly increasing during CO. HR was not affected by PC, but increased during CO. After PC and CO were stopped, the parameters tended to return to the values observed before the respective interventions (see also Electronic Supplementary Material). A slight increase in MAP, DBP, TPR, but no significant changes in the other parameters measured were observed during the control test. The gain in MAP and DBP in the control test was smaller than that in the main test (both $p<0.01$ ), while the increase in the TPR was similar between the two tests $(p=0.19)$. There was no difference in self-reported leg pain between the main and control tests $(3.5 \pm 1.9$ vs. $3.6 \pm 1.8$ on a scale of $10 ; p=0.72)$. The rating of perceived exertion in the main test during PC was $6.3 \pm 1.1$ (range $6-20$ points).

\section{Discussion}

The aim of this study was to develop a new, safe, simple and automated method for testing mechanoreflex-metaboreflex interactions in humans. We hypothesized that (1) PC alone affects hemodynamic and ventilatory parameters and, in particular, increases blood pressure, SV and MV (mechanoreflex activation); (2) adding $\mathrm{CO}$ to PC leads to additional changes, such as additional increases in blood pressure and MV (metaboreflex activation); and (3) once PC and CO cease, these parameters tend to return to the values observed before the aforementioned interventions. The results of this study support our hypotheses. Therefore, for the first time, we present a novel protocol that is effective in stimulating mechano- and metaboreceptors simultaneously and physiologically in a single experiment, using commercially available equipment.

The first step of this study was to choose the optimal technique to stimulate the mechanoreflex. Various methods have been used in earlier studies, such as involuntary muscle contractions with the use of electrostimulation $[9,16]$; muscle stretching performed by investigators $[17,19]$ or by applying custom-built devices [18, 20]; passive movements on tandem bicycle [15] or on custom-built equipment and
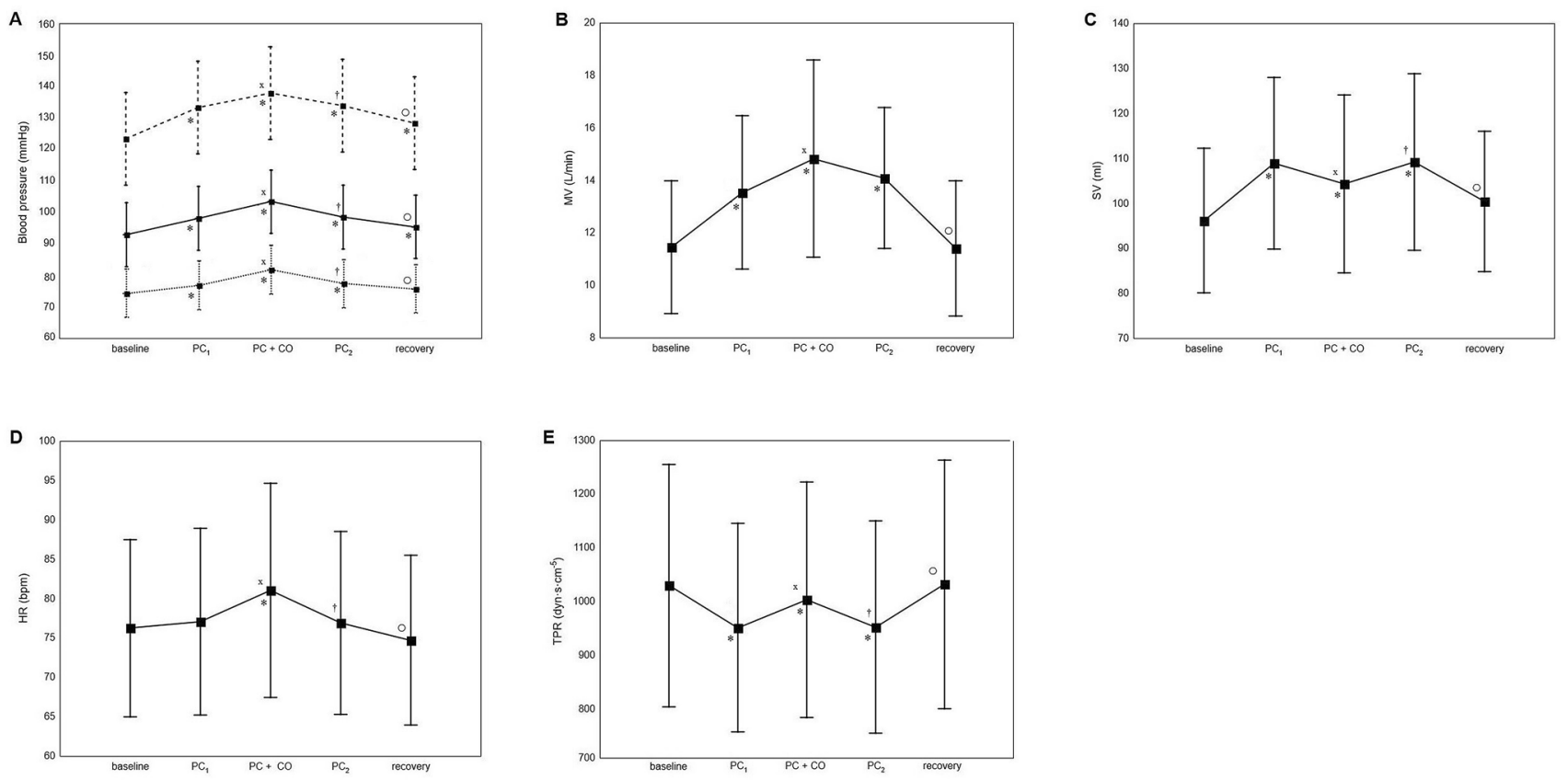

Fig. 4 Plots showing the mean values and standard deviations of the mean arterial pressure (MAP; a, solid line), systolic blood pressure $(S B P$; a, dashed line), diastolic blood pressure ( $D B P$; a, dotted line), minute ventilation $(M V ; \mathbf{b})$, stroke volume $(S V ; \mathbf{c})$, heart rate $(H R ; \mathbf{d})$ and total peripheral resistance (TPR; $\mathbf{e})$ in consecutive test periods of the main test. See caption to Fig. 1 for description of the phases of the main test: baseline, $\mathrm{PC}_{1}, \mathrm{PC}+\mathrm{CO}, \mathrm{PC}_{2}$, recovery period. Changes in each parameter are statistically significant according to repeated measures analysis of variance when all test periods are considered together. ${ }^{*} p<0.05$ between baseline and given period, ${ }^{x_{p}} p<0.05$ between $\mathrm{PC}_{1}$ and $\mathrm{PC}+\mathrm{CO},{ }^{\dagger} p<0.05$ between $\mathrm{PC}+\mathrm{CO}$ and $\mathrm{PC}_{2}$, ${ }^{\circ} p<0.05$ between $\mathrm{PC}_{2}$ and $\mathrm{PC}+\mathrm{CO}$ 
cycle ergometers $[8,14]$. All these methods were designed to reduce the possibility of metabolite accumulation, which can stimulate metaboreceptors, and to eliminate voluntary movements that engage central command. However, electrostimulation and muscle stretching appear to be less reliable than passive movement. The major concern with using electrostimulation is the potentially confounding effect of concomitant activation of muscle metaboreceptors, as it has been shown that electrostimulation induces metabolite production [21-23]. This effect may explain the highly discrepant observations across studies targeting small muscles (slight increase in MAP, no effect on HR) [9] and large muscles (large increase in MAP and HR) [16]. The effects of muscle stretching are also difficult to interpret, given that stretching and contracting muscles stimulate different types of mechanoreceptors [1]. Moreover, stretching does not mimic the physiological model of dynamic exercise.

The results of the few studies that have used stretching to stimulate mechanoreceptors are highly variable. Depending on the type of a muscle, stretching induced an increase in MAP without affecting HR (in agreement with our findings) [20] or a decrease in MAP with an increase in HR [17]. The latter result is inconsistent with those of other studies reporting that mechanoreceptor stimulation induces an increase in MAP $[9,14-16,20,29]$. Taking these concerns into account, we decided to choose PC to induce the mechanoreflex. This technique was first used by Nobrega et al. [29] and caused elevations in MAP and SV. Similar results were observed in all subsequent experiments involving PC, regardless of whether a tandem bicycle [15] or ergometer was adapted [14]. The technique has also been shown to be applicable for patients with heart failure, in whom it evoked MV increases [26].

Our results confirm that PC stimulates mechanoreceptors, which-most notably-increased SBP (8\%; from $120 \pm 14 \mathrm{mmHg}$ at baseline to $130 \pm 14 \mathrm{mmHg}$ at PC), SV (13\%; from $96 \pm 16 \mathrm{~mL}$ at baseline to $109 \pm 19 \mathrm{~mL}$ at PC) and MV (17\%; from $11 \pm 2 \mathrm{~L} / \mathrm{min}$ at baseline to $13 \pm 3 \mathrm{~L} /$ min at PC). All parameters decreased after PC was stopped, which suggests that hemodynamic and ventilatory changes persist as long as the mechanoreceptors are stimulated. We used a fully automated and commercially available, adjustable cycle ergometer, originally designed for rehabilitation for paresis and paralysis in lower extremities; it may become the standard device for testing the mechanoreflex.

The second step of this study was to create a valuable method for testing mechanoreflex-metaboreflex interactions in humans. Virtually all protocols used in previous studies to test interactions between mechanoreceptors and metaboreceptors involved muscle stretching [17-19, 24] in the following order of tasks: (1) exercise was performed, (2) post-exercise $\mathrm{CO}$ was applied and (3) the muscle was stretched. Aside from the issues with muscle stretching mentioned before, this approach also does not engage both reflexes in a physiological and simultaneous manner. Rather, it creates an interim metabolic background that overlaps with stretching. Our protocol implements a completely different paradigm. First, we used PC, which is effective in stimulating isolated mechanoreceptors and evokes consistent cardiovascular and ventilatory effects; second, we introduced a different order of interventions, wherein $\mathrm{CO}$ is applied on the limbs that are being moved passively. This method imitates the physiological stimulation of particular receptors during physical activity. In general, the first response comes from mechanoreceptors, and then, when metabolites accumulate, the metaboreflex starts to induce hemodynamic changes $[1,7]$.

Previous studies on mechanoreflex-metaboreflex interactions yielded inconsistent results, even when the same methods and muscles were used. Stretching of the calf muscle during $\mathrm{CO}$ in one study resulted in an increase in SV and HR without any change in MAP [18], whereas in another study it induced only a small increase in DBP [19]. In yet another study, isolated wrist stretching did not result in any changes in hemodynamic parameters, with only a small increase in MAP occurring when $\mathrm{CO}$ was added to stretching [24]. Using our protocol, we observed that almost all of the tested parameters showed regular, characteristic patterns in response to $\mathrm{PC}$ and $\mathrm{CO}$. Additional and significant increases in blood pressure and $\mathrm{MV}$ were induced by $\mathrm{CO}$ during PC, indicating that the stimulation of metaboreceptors resulted in an additional response. These observations suggest our new protocol is a reliable tool for testing mechanoreflex-metaboreflex interactions in humans, with greater validity than protocols based on muscle stretching.

Interestingly, we observed a pattern of changes in HR that suggests hyperadditive interactions between the metabo- and mechanoreflex. Specifically, HR was not affected by PC or CO separately, but it increased when $\mathrm{CO}$ was added to PC. A lack of HR response to PC has been shown previously [14, 29]. Similarly, the isolated stimulation of metaboreceptors does not produce essential increases in HR [7]. The potential influence of the metaboreflex on HR is masked, even during $\mathrm{CO}$, by parasympathetic reactivation, mainly due to a loss of central command [30-32]. Barbosa et al. [33] showed that blockade of the exercise pressor reflex attenuates the HR response to active cycling, which indicates that the increase in HR is also not caused solely by central command. Considering these facts and the results of our study, we can conclude that the aforementioned increase in HR is probably a unique example of cooperation between the mechanoreflex and metaboreflex. Thus, to evoke a substantial increase in HR, both mechanoreceptors and metaboreceptors must be stimulated. The aggregate feedback from both types of receptors is essential to provide a sufficient stimulus that meets the threshold for a change in HR. 
Finally, the exaggerated exercise pressor reflex in heart failure and hypertension is presumed to be a relevant component of autonomic dysfunction, which is characteristic of these disorders [1,2, 8-11]. Regarding the global character of these conditions, it is extremely important to comprehensively understand the precise mechanisms of deterioration in the metabo- and mechanoreflex. This knowledge may enable development of targeted therapies for cardiovascular diseases, based on selective blockade of the components of the exercise pressor reflex [34] and specific exercise training [1]. Thus, introducing a new reliable method for testing the metabo- and mechanoreflex is essential for clinical purposes as well.

\section{Limitations}

It is difficult to eliminate voluntary muscle contractions when an individual's legs are being moved by an ergometer. To prevent this disturbance and stabilize subjects' calves, we use rails connected to the ergometer pedals (Fig. 2). The rails are standard parts of the ergometer. The subjects are also asked to relax, not to perform any movements and not to think about exercise. We did not notice any voluntary movements in the subjects throughout the protocol, and the mean physical effort rating on the Borg scale was very low. However, electromyography signals from the leg muscles were not recorded and thus central command contribution cannot be entirely excluded. We attributed the slight increase in MAP, DBP, and TPR in the control test to the modest accumulation of metabolites at rest and stimulation of metaboreceptors, although other factors cannot be excluded. It is unlikely that these changes were caused by pain, since we found no difference between the severity of pain in the limb caused by $\mathrm{CO}$ between the main and control tests.

\section{Conclusions}

Our protocol has, for the first time, introduced the application of $\mathrm{CO}$ during $\mathrm{PC}$, which allows us to mimic a physiological model of dynamic exercise. With the use of this scheme, we confirmed our hypothesis that PC evokes increases in blood pressure, SV and MV (mechanoreflex activation); adding $\mathrm{CO}$ to $\mathrm{PC}$ induces additional increases in blood pressure and MV (metaboreflex activation); and these parameters return to the values observed before $\mathrm{PC}$ and $\mathrm{CO}$ after these interventions are stopped. Our model has several advantages since it involves a safe, simple and automated method of stimulating mechanoreceptors in a physiological way and, additionally, stimulating metaboreceptors without terminating mechanoreceptor activation. We believe that this method will provide new opportunities to study interactions between these two components of the exercise pressor reflex, which is an intriguing area of scientific research but remains to be fully elucidated.

Acknowledgements This research was financially supported by the Ministry of Science and Higher Education (Poland)/Wroclaw Medical University, internal number: SUB.A090.19.035.

Author contributions $\mathrm{AL}, \mathrm{BP}, \mathrm{W} \mathrm{L}, \mathrm{MFP}$ and $\mathrm{BP}$ conceived and designed the study; $\mathrm{AL}$ and $\mathrm{W} €$ collected the data; $\mathrm{AL}$ wrote the first draft the manuscript; AL, BP, WŁ, MFP and BP checked and corrected manuscript. All authors read and approved the final draft of the manuscript.

\section{Compliance with ethical standards}

Conflict of interest The authors declare that they have no conflict of interest.

Open Access This article is licensed under a Creative Commons Attribution 4.0 International License, which permits use, sharing, adaptation, distribution and reproduction in any medium or format, as long as you give appropriate credit to the original author(s) and the source, provide a link to the Creative Commons licence, and indicate if changes were made. The images or other third party material in this article are included in the article's Creative Commons licence, unless indicated otherwise in a credit line to the material. If material is not included in the article's Creative Commons licence and your intended use is not permitted by statutory regulation or exceeds the permitted use, you will need to obtain permission directly from the copyright holder. To view a copy of this licence, visit http://creativecommons.org/licenses/by/4.0/.

\section{References}

1. Murphy MN, Mizuno M, Mitchell JH, Smith SA (2011) Cardiovascular regulation by skeletal muscle reflexes in health and disease. Am J Physiol Hear Circ Physiol 301:H1191-204. https://doi. org/10.1152/ajpheart.00208.2011

2. Smith SA, Mitchell JH, Garry MG (2006) The mammalian exercise pressor reflex in health and disease. Exp Physiol 91:89-102. https://doi.org/10.1113/expphysiol.2005.032367

3. Kaufman MP, Hayes SG (2002) The exercise pressor reflex. Clin Auton Res 12:429-439. https://doi.org/10.1007/s1028 6-002-0059-1

4. Amann M, Blain GM, Proctor LT et al (2011) Implications of group III and IV muscle afferents for high-intensity endurance exercise performance in humans. J Physiol 589:5299-5309. https ://doi.org/10.1113/jphysiol.2011.213769

5. Kaufman MP (2012) The exercise pressor reflex in animals. Exp Physiol 97:51-58. https://doi.org/10.1113/expphysiol.2011.05753 9

6. Secher NH, Amann M (2012) Human investigations into the exercise pressor reflex. Exp Physiol 97:59-69. https://doi.org/10.1113/ expphysiol.2011.057679

7. Nobrega ACL, Leary DO, Silva BM et al (2014) Neural regulation of cardiovascular response to exercise: role of central command and peripheral afferents. Biomed Res Int 2014:1-20. https://doi. org/10.1155/2014/478965

8. Ives SJ, Amann M, Venturelli M et al (2016) The mechanoreflex and hemodynamic response to passive leg movement in 
heart failure. Med Sci Sports Exerc 48:368-376. https://doi. org/10.1249/MSS.0000000000000782

9. Middlekauff HR, Nitzsche EU, Hoh CK et al (2001) Exaggerated muscle mechanoreflex control of reflex renal vasoconstriction in heart failure. J Appl Physiol 90:1714-1719. https://doi. org/10.1152/jappl.2001.90.5.1714

10. Piepoli M, Clark AL, Volterrani M et al (1996) Contribution of muscle afferents to the hemodynamic, autonomic, and ventilatory responses to exercise in patients with chronic heart failure: effects of physical training. Circulation 1:940-952

11. Ponikowski PP, Chua TP, Francis DP et al (2001) Muscle ergoreceptor overactivity reflects deterioration in clinical status and cardiorespiratory reflex control in chronic heart failure. Circulation 104:2324-2330. https://doi.org/10.1161/hc4401.098491

12. Schmidt H, Francis DP, Rauchhaus M et al (2005) Chemo- and ergoreflexes in health, disease and ageing. Int J Cardiol 98:369378. https://doi.org/10.1016/j.ijcard.2004.01.002

13. Houssiere A, Najem B, Pathak A et al (2006) Chemoreflex and metaboreflex responses to static hypoxic exercise in aging humans. Med Sci Sports Exerc 38:305-312. https://doi.org/10.1249/01. mss.0000187426.93464.81

14. Krzemiński K, Kruk B, Nazar K et al (2000) Cardiovascular, metabolic and plasma catecholamine responses to passive and active exercises. J Physiol Pharmacol 51:267-278

15. Vorluni L, Volianitis S (2008) Baroreflex control of sinus node during dynamic exercise in humans: effect of muscle mechanoreflex. Acta Physiol 192:351-357. https://doi.org/10.111 1/j.1748-1716.2007.01766.x

16. Hureau TJ, Weavil JC, Thurston TS et al (2018) Identifying the role of group III/IV muscle afferents in the carotid baroreflex control of mean arterial pressure and heart rate during exercise. $\mathrm{J}$ Physiol. https://doi.org/10.1113/JP275465

17. Venturelli M, Cè E, Limonta E et al (2017) Central and peripheral responses to static and dynamic stretch of skeletal muscle: mechano- and metaboreflex implications. J Appl Physiol 122:112-120. https://doi.org/10.1152/japplphysiol.00721.2016

18. White MJ, Lykidis CK, Balanos GM (2013) The pulmonary vascular response to combined activation of the muscle metaboreflex and mechanoreflex. Exp Physiol 98:758-767. https://doi. org/10.1113/expphysiol.2012.068528

19. Fisher JP, Bell MPD, White MJ (2005) Cardiovascular responses to human calf muscle stretch during varying levels of muscle metaboreflex activation. Exp Physiol 90:773-781. https://doi. org/10.1113/expphysiol.2005.030577

20. Choi H-M, Stebbins CL, Lee O-T et al (2013) Augmentation of the exercise pressor reflex in prehypertension: roles of the muscle metaboreflex and mechanoreflex. Appl Physiol Nutr Metab 38:209-215. https://doi.org/10.1139/apnm-2012-0143

21. Vanderthommen M, Duteil S, Wary C et al (2003) A comparison of voluntary and electrically induced contractions by interleaved
1H- and 31P-NMRS in humans. J Appl Physiol 94:1012-1024. https://doi.org/10.1152/japplphysiol.00887.2001

22. Vanderthommen M, Duchateau J (2007) Electrical stimulation as a modality to improve performance of the neuromuscular system. Exerc Sport Sci Rev 35:180-185. https://doi.org/10.1097/ jes.0b013e318156e785

23. McNeil CJ, Murray BJ, Rice CL (2006) Differential changes in muscle oxygenation between voluntary and stimulated isometric fatigue of human dorsiflexors. J Appl Physiol 100:890-895. https ://doi.org/10.1152/japplphysiol.00921.2005

24. Cui J, Mascarenhas V, Moradkhan R et al (2007) Effects of muscle metabolites on responses of muscle sympathetic nerve activity to mechanoreceptor(s) stimulation in healthy humans. AJP Regul Integr Comp Physiol 294:R458-R466. https://doi.org/10.1152/ ajpregu.00475.2007

25. Penáz J (1992) Criteria for set point estimation in the volume clamp method of blood pressure measurement. Physiol Res 41:5-10

26. Scott AC, Francis DP, Ceri Davies L et al (2000) Contribution of skeletal muscle "ergoreceptors" in the human leg to respiratory control in chronic heart failure. J Physiol 529:863-870. https:// doi.org/10.1111/j.1469-7793.2000.00863.x

27. Magder S (2018) The meaning of blood pressure. Crit Care 22:110. https://doi.org/10.1186/s13054-018-2171-1

28. Borg G (1982) Psychophysical bases of perceived exertion. Med Sci Sport Exerc 14:377-381

29. Nóbrega ACL, Williamson JW, Friedman DB et al (1994) Cardiovascular responses to active and passive cycling movements. Med Sci Sports Exerc 26:709-714. https://doi.org/10.1249/00005 768-199406000-00009

30. Fisher JP, Seifert T, Hartwich D et al (2010) Autonomic control of heart rate by metabolically sensitive skeletal muscle afferents in humans. J Physiol 588:1117-1127. https://doi.org/10.1113/jphys iol.2009.185470

31. Crisafulli A, Piras F, Filippi M et al (2011) Role of heart rate and stroke volume during muscle metaboreflex-induced cardiac output increase: differences between activation during and after exercise. J Physiol Sci 61:385-394. https://doi.org/10.1007/s1257 6-011-0163-x

32. Alam M, Smirk FH (1937) Observations in man upon a blood pressure raising reflex arising from the voluntary muscles. J Physiol 89:372-383

33. Barbosa TC, Vianna LC, Fernandes IA et al (2016) Intrathecal fentanyl abolishes the exaggerated blood pressure response to cycling in hypertensive men. J Physiol 594:715-725. https://doi. org/10.1113/JP271335

34. Smith SA, Mitchell JH, Naseem RH, Garry MG (2005) Mechanoreflex mediates the exaggerated exercise pressor reflex in heart failure. Circulation 112:2293-2300. https://doi.org/10.1161/ CIRCULATIONAHA.105.566745 